\title{
Numerical simulation of star formation in filamentary dark molecular clouds
}

\author{
Pak Shing $\mathbf{L i}^{1}$, Richard I. Klein ${ }^{1,2}$ and Christopher F. McKee ${ }^{1,3}$ \\ ${ }^{1}$ Astronomy Department, University of California, \\ 501 Campbell Hall \#3411, Berkeley, CA 94720-3411, USA \\ email: psli@berkeley.edu \\ ${ }^{2}$ Lawrence Livermore National Laboratory, \\ P.O. Box 808, L-23, Livermore, CA 94550, USA, \\ email: klein@astron.berkeley.edu \\ ${ }^{3}$ Physics Department, University of California, \\ Berkeley, CA 94720, USA, \\ email: cmckee@astro.berkeley.edu
}

\begin{abstract}
Numerical simulations of star formation faces challenges including the huge spatial dynamic range and the presence of multiply coupled highly non-linear physics, such as magnetic field, supersonic turbulence, gravitation, radiation and protostellar outflow feedback. We present in this symposium our latest high resolution adaptive mesh refinement numerical simulations of the formation of filamentary dark molecular clouds from $4.55 \mathrm{pc}$ size scale down to the formation of a protostellar cluster with maximum resolution at $28 \mathrm{AU}$. The physical properties of the long braided filamentary dark cloud formed in the simulation, the magnetic field properties of the cloud clumps, and the protostellar mass function in the simulations match well with the latest observations.
\end{abstract}

Keywords. Magnetic fields-MHD-ISM: magnetic fields-ISM: kinematics and dynamicsstars: formation

\section{Introduction}

Observations show that the star formation process starts from the giant molecular cloud (GMC) complex of several tens of parsecs (pcs) all the way down to the formation protostars at the size scale of solar radius (9 orders of magnitude in spatial scale). In between, we observe long filamentary dark clouds of a few to more than $10 \mathrm{pc}$ in length, large molecular cloud clumps about 0.1 to $1 \mathrm{pc}$ in size, dense cloud cores less than $0.1 \mathrm{pc}$ which house protostellar formation, and protostellar accretion disks $\leqslant 1,000 \mathrm{AU}$. Multiply coupled highly non-linear physics are involved in the process, including magnetic field, supersonic turbulence, gravity, radiation and protostellar outflow feedback. It is a challenge to simulate the entire process in a single numerical simulation. To achieve such a long term challenge, we need efficient adaptive codes, such as adaptive mesh refinement (AMR) code, that can manage memory usage efficiently and are highly scalable on largescale high performance supercomputers. Here we present some of the highlights of our simulation results using our radiative-magnetohydrodynamics AMR code ORION2 (Li et al. 2012) on formation of filamentary dark clouds, the magnetic properties of the dense cloud clumps, and protostellar cluster formation. 


\section{Simulations}

Our simulation starts with a large scale turbulence region with 3D thermal Mach number of 10 . Using line width-size relation, the length of the periodic cubical region is $4.55 \mathrm{pc}$. We use the mean density of the GMC at such size scale and the total mass of the whole simulation region is $3110 M_{\odot}$. Motivated by observations, the other two dimensionless parameters we chose are the Alfvénic Mach number $\left(\mathcal{M}_{\mathrm{A}}\right)$ and the virial parameter, both of which are unity. We assume isothermal conditions and ideal MHD. The corresponding plasma $\beta=0.02$ and the mass-to flux ratio is 1.62 . This corresponds to a relatively strong initial magnetic field that is found to be more appropriate in describing the magnetic field properties of molecular clouds than our other model with exactly the same initial conditions ( $\mathrm{Li}$ et al. 2015a) but 10 times weaker initial field strength. The base grid is $512^{3}$ with two levels of grid refinement. We drive the system using a solenoidal field for two crossing times, defined as the box length divided by the rms velocity. At this time the system has reached a turbulent equilibrium state. We turn on gravity and continue driving the system with constant energy injection to maintain a global $3 \mathrm{D}$ value of Mach 10. The simulation continues for $2 / 3$ of a free-fall time $\left(1 t_{\mathrm{ff}}=1.4 \times 10^{6} \mathrm{yrs}\right)$. We use this simulation to study the formation of filamentary IRDCs.

To study the formation of a protostellar cluster, we restart the IRDCs simulation at about $0.25 t_{\mathrm{ff}}$ when no cell has violated the Jeans condition yet. We allow maximum refinement to 6 levels in a specified $4.2 \mathrm{pc}^{3}$ zoom-in region that encompasses the main filament to make the zoom-in simulation computationally feasible. We attain a maximum 28 AU resolution in this zoom-in region. Anywhere outside this region will be simulated at the base level resolution. The protostellar star particles used in the simulation, evolve based on subgrid model of protostars. They are created at the highest level of grid refinement. As a result, no protostar is created outside the zoom-in region. Nevertheless, more than $75 \%$ of the most massive clumps exist in the zoom-in region. In the zoom-in simulation, we include radiation transfer and protostellar outflow feedback to study protostellar cluster formation.

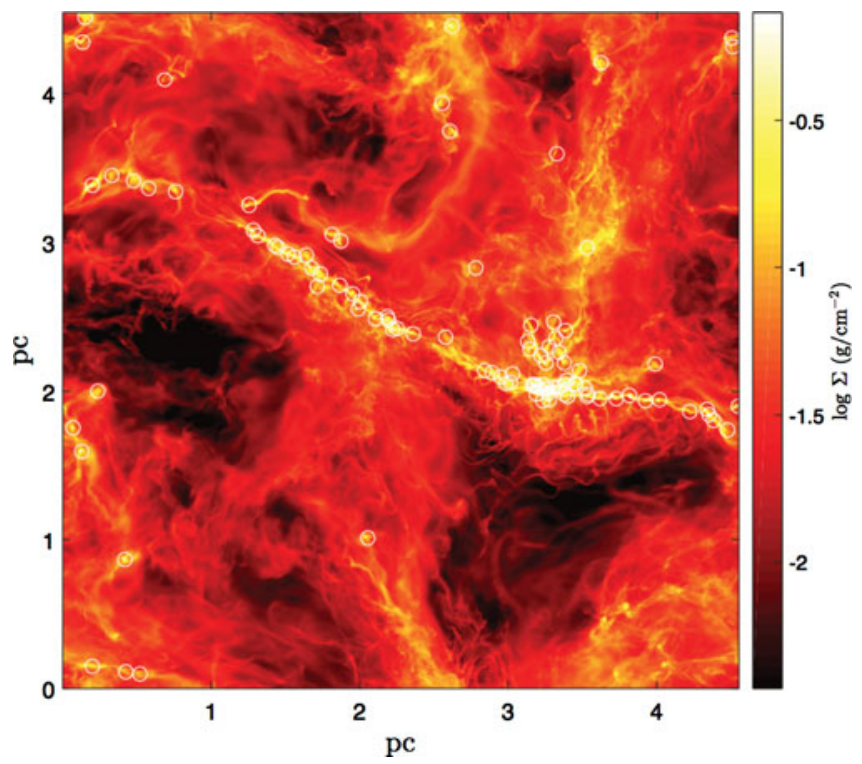

Figure 1. Column density map of the simulated region showing the main long massive filament cloud near the center and the 100 most massive clumps (white circles).

\section{Highlights of the simulation results}

Here we discuss three highlights from our simulations. In Fig. 1, we show the column density map of the entire region along the mean field direction from the IRDCs simulation 
at $0.5 t_{\mathrm{ff}}$. A dense and long filamentary cloud is located near the center. The filament is $\sim 4.5 \mathrm{pc}$ long and has a mass of $\sim 660 M_{\odot}$. The volume and column densities are about $2 \times 10^{4} \mathrm{~cm}^{-3}$ and $4 \times 10^{22} \mathrm{~cm}^{-2}$, similar to some observed IRDCs. The mass per unit length is $\sim 77.6 \mathrm{M}_{\odot} \mathrm{pc}^{-1}$ within 0.2 pc radius from the filament axis, about five times of the critical density $2 c_{s}^{2} / G$ and gravitationally bounded. In the map, we plot the 100 most massive clumps marked by the white circles. Most of them are along the main filament. Note the appearance of another filament near the upper right connected to the main filament. The two filaments are in fact colliding. This config-

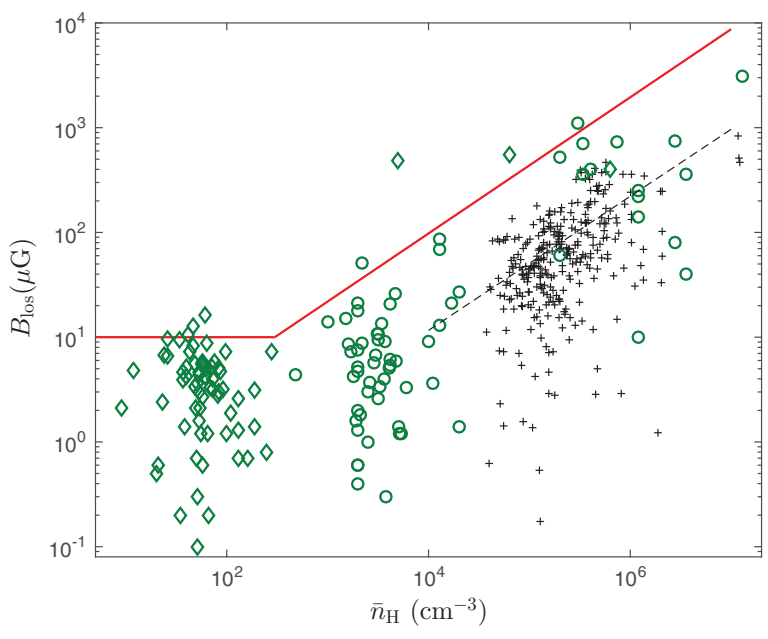

Figure 2. $B_{\text {los }}$-n relation of Zeeman observations from Crutcher et al. (2010) (HI green diamond, $\mathrm{OH}+\mathrm{CN}$ green circles) and the 100 most massive clumps (black plus) from the initially strong magnetic field simulation. The dashed line is the best power law fit to the simulation data. uration is strikingly similar in appearance to the dark cloud SDC13 (Peretto et al. 2014). The comparison with SDC13 will be reported in Li et al. (2016).

In Li et al. (2015a), we have performed a detail statistical comparison of the magnetic field properties of the 100 most massive clumps with the Zeeman observations of molecular clouds summarized in Crutcher et al. (2010). In Fig. 2, we plot the B-n relation of the observed line-of-sight (los) magnetic field and volume density of the HI, OH, and CN sources with the 100 most massive clumps from the IRDCs simulation. The distribution of clumps from simulation resembles the power-law-like B-n relation of the molecular $\mathrm{OH}$ and $\mathrm{CN}$ sources well. In fact, the B-n relation of the total magnetic field from the simulation is consistent with $B_{\text {tot }} \propto n^{0.65}$ concluded in Crutcher et al. (2010). The large scattering in the observations is the result of dispersion in total field strength and the line of sight projection effect. In another simulation with initially 10 times weaker field, the B-n relation of the clumps is very similar to the initially strong field simulation and visually indistinguishable in the $B_{\text {los }}$ vs $n$ plot. It is because of the field amplification in the weak field model due to turbulent driving. Despite the similarity in the B-n relation between the two simulations, there is a clear difference in the distribution of field orientation of the clumps with respect to the mean field direction, as shown in Fig. 3. The distribution from the initially strong field simulation is consistent with the recent observational study of molecular clouds

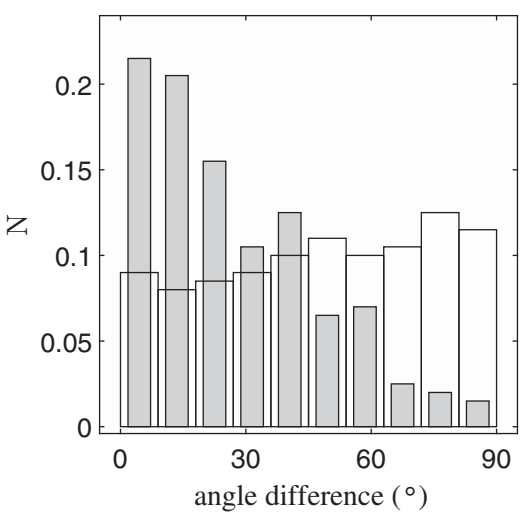

Figure 3. Distribution of magnetic field orientation of the 100 most massive clumps, w.r.t. the mean field direction, of the initially strong (open) and weak (shaded) field simulations. magnetic field orientation by Li et al. (2009). For the initially weak field simulation, the 

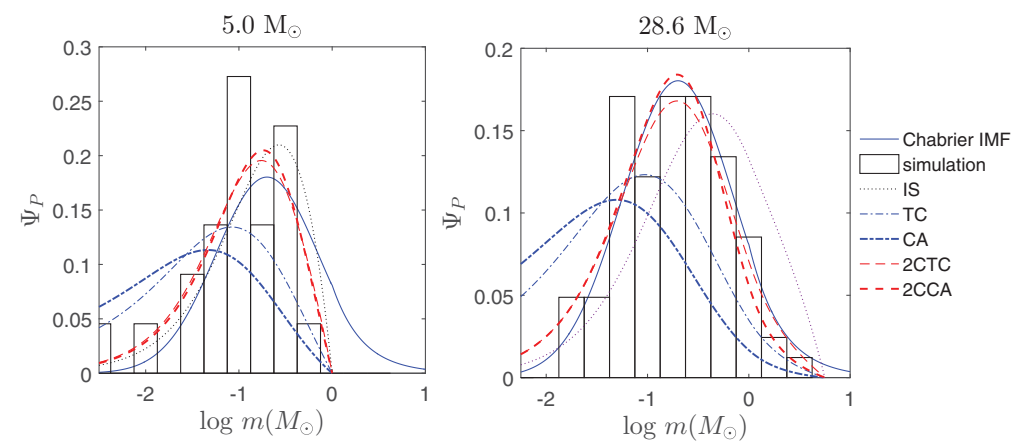

Figure 4. The PMFs at the time when the total masses of the protostars are 5 and $28.6 M_{\odot}$ with 4 theoretical PMF models and the Chabrier IMF. The 2CTC model best fits the PMF from the simulation.

distribution is basically random which is not consistent with the strong alignment of the global B field of the surrounding intercloud medium.

In the 6-level zoom-in simulation, at the time of $0.5 t_{\mathrm{ff}}$, there are total 82 protostellar particles formed. Fig. 4 shows the protostellar mass functions (PMFs) of the protostars when the total masses of protostars are $5 M_{\odot}$ and $28.6 M_{\odot}$ (at the end of the simulation), compared with several theoretical PMFs (McKee \& Offner 2010). Using a reduced $\chi^{2}$ comparison, the two-component turbulent core PMF best fits the simulation results for all time. At the end of the simulation, the PMF also matches the Chabrier stellar initial mass function (IMF) (Chabrier 2005) very well. The median of the PMF is $0.196 M_{\odot}$, very close to the expected $0.2 M_{\odot}$ from Chabrier IMF. The results of the zoom-in simulation will be reported in Li et al. (2015b).

\section{Conclusions}

Using AMR combined with a zoom-in method, we achieve more than 4 orders of magnitude in spatial resolution dynamic range in these star formation simulations. We successfully recreate many observational results of the star formation process including the large scale structure and dynamics of filamentary IRDCs, the magnetic field properties of cloud clumps, and the formation of the protostellar cluster. New simulations with an even larger dynamic range of spatial resolution of 6 orders of magnitude are undertaken in order to continue the simulation down to scales of the formation of protostellar accretion disks. Multiphysics AMR simulations are crucial in following star formation from large scale clouds to protostellar formation.

\section{References}

Chabrier, G. 2005, in: E. Corbelli \& F. Palla (eds.), The Initial Mass Function 50 Years Later Ap\&SSL Conf. Ser., 327, 41

Crutcher R. M., Wandelt B., Heiles C., Falgarone E., \& Troland T. H. 2010, ApJ, 725, 466

Li H.-b., Dowell C. D., Goodman A., Hildebrand R., \& Novak, G. 2009, ApJ, 704, 891

Li, P. S., Martin, D. F., Klein, R. I., \& McKee, C. F. 2012, ApJ, 745, 139

Li, P. S., McKee, C. F., \& Klein, R. I. 2015a, MNRAS, 30, 490

Li, P. S., Klein, R. I., \& McKee, C. F. 2015b, in preparation

Li, P. S., Klein, R. I., \& McKee, C. F. 2016, in preparation

McKee, C. F. \& Offner, S. S. R.. 2010, ApJ, 716, 167

Peretto, N., Fuller, G. A., André, Ph., et al. 2014, A\&ऽA, 561, A83 